\title{
THE ADVENT OF COSMOLOGICAL GAS DYNAMIC SIMULATIONS
}

\author{
August E. Evrard \\ Institute of Astronomy, Madingley Road, Cambridge CB3 0HA, UK
}

The next move forward in simulations of cosmological structure is to include the hydrodynamics and thermal history of a gaseous component. The task is not an easy one. The dynamic range is wide in all interesting quantities (density, temperature, length-scales, time-scales, etc.). Generic initial mass distributions sampled from Gaussian random fields will, for many interesting power spectra, lead to a high degree of substructure present at all stages of the evolution. Grid-based hydrodynamic techniques currently lack the resolution necessary to evolve several levels of a clustering hierarchy simultaneously . A particle-based method known as SPH (Smoothed Particle Hydrodynamics, see Monoghan (1985) for a review) appears best suited for cosmological application. I have recently imbedded the technique into the P3M N-body code, described by Efstathiou et al. (1985) and used extensively by Efstathiou and collaborators, most recently in investigations of the cold dark matter scenario.

The gas is represented by a set of particles carrying local thermodynamic information along with the usual kinematic data. Other collisionless components (dark matter, galaxies) are represented by additional species of particles. SPH employs direct interpolation from neighboring particles to obtain estimates of local gas densities, pressure gradients, flow convergence, etc.. The equations of motion for gas element $i$ are written as a sum of pairwise interactions with neighboring particles $j$. These interactions have a finite range $h$ (the range of the interpolation kernel) which is allowed to vary in both space and time, essentially scaling with the local interparticle separation. Thus, a wide dynamic range in density is achieved. An adiabatic equation of state with ratio of specific heats $\gamma$ is assumed, alternative equations of state are readily implemented. Shock heating is incoporated via an artificial viscosity. Radiative cooling, specified by an input function dependent on local gas density and temperature, has also been included. Details and tests of the method can be found in Evrard (1987).

An initial application of the method to the evolution of gas in rich clusters of galaxies is currently under way. Addition of ad hoc star formation, with associated energy and metallicity feedback, will enable detailed study of the galaxy formation problem.

Efstathiou, G., Davis, M., Frenk, C. and White, S. 1985, Ap. J. Supl., 57, 241. Evrard, A.E. 1987, in preparation.

Monaghan, J.J. 1985 Comp. Phys. Reports, 3, 71. 